\title{
Blended Churn Predictive System for Quadruple-Patterned Churn Classification Towards Effective Customer Behavioural Management
}

\author{
Ayodeji Ibitoye ${ }^{1}$, Olufade Onifade ${ }^{1,2}$ \\ ${ }^{1}$ Computer Science Department, Bowen University, Iwo, Nigeria \\ ${ }^{2}$ Computer Science Department, University of Ibadan, Ibadan, Nigeria
}

Email address:

Ibitoye_ayodeji@yahoo.com (A. Ibitoye), fadowilly@yahoo.com (O. Onifade)

\section{To cite this article:}

Ayodeji Ibitoye, Olufade Onifade. Blended Churn Predictive System for Quadruple-Patterned Churn Classification Towards Effective Customer Behavioural Management. International Journal on Data Science and Technology. Vol. 6, No. 2, 2020, pp. 56-59.

doi: $10.11648 /$ j.ijdst.20200602.12

Received: June 17, 2020; Accepted: July 2, 2020; Published: August 20, 2020

\begin{abstract}
The adoption of product centric approach to customer acquisition by many subscriber based companies has become a factor, which influences customer misclassification in existing churn predictive models. While the transaction volume, velocity, and varieties for basic churn processes continues to increase exponentially, every customer remained a potential churner to a certain degree. Although, existing churn prediction models classifies customers as churner or nonchurner, many of its approaches assign equal weight to features while the customer's power of influence from sociotransactional data mining are neglected in churn behaviour management. Here, the developed Churn Predictive System is a composite of Recency-Frequency-Monetary-Influence model through customer segmentation management and Fuzzy-Weighed Feature Engineering model, which trained and tested transactional records using Random Forest and Adaboost Ensemble Learning in a 5-fold cross validation protocol. This System was coupled (Customer Segmentation + Ensemble Learning) to achieve a quadrupled customer's churn category as Churner, Potential Churner, Inertia Customer and Premium Customers. The results from the developed system juxtapose the need for a new approach to churn prediction in customer behavioural management.
\end{abstract}

Keywords: Churn Prediction, Fuzzy-weight, Ensemble Machine Learning, Customer Segmentation, Customers' Behavioural Management

\section{Introduction}

In modern business world, the risk of losing customers to another service provider is so enormous especially for subscriber based organizations. Although, in competitive business cycle where advertisement and promotional offers are used for customers' acquisition, customer churn seems unnoticeable in bits per day, per week [1]. However, when compounded over a period; say a quarter or over a year, a business could be losing a fortune of their customers to competitors. Consequently, instead of using the traditional approach to detect customer churn, machine learning models [2] have been adopted by various researchers to find patterns and relationships in large amount of data. The data, which represents different customers' behavioural traits remained an important component for effective customer retention.

Thus, as the need for customers' acquisition and retention became a top concern for organizations; aside, machine learning techniques through single or ensemble methods, market/customer segmentation technique have also been used to identify and extract, which parts of the service a customer is using, and how often the customer uses such. The behavioural data through these methods are represented as attributes or features that are manipulated to define a customer and their churn prediction category [3]. While existing Recency-Frequency-Monetary model through customer segmentation neglects the customer's power of influence in community based churn prediction, existing methods in churn prediction via machine learning also assign equal weights to features. This processes as increasing led to 
churn misclassification, and less effective targeted decision support in acquiring new customers or retaining existing once. Therefore, in section two, related works in customers' segmentation and machine learning patterns are presented. In section three, the developed blended churn predictive system is presented. Sample experiments and evaluations are documented in section four (4) while the research is concluded in section five (5).

\section{Related Works}

One of the most imperative service aspects in telecommunications industry among other subscriber based organization is churn prediction. It is also an important factor to consider for Customer Relationship Management (CRM) [4]. Churn prediction has become significantly necessary because a business is lost when it cost more to acquire a customer then retaining existing once. Therefore, to measure customers' loyalty for decision support through customer retention, churn prediction algorithms became necessary. While different customers exhibits various behavioural attributes, the quality of a data pre-processing and analyses contributes to churn prediction. Thus, due to the importance of attributes is churn prediction, [5] provided an overview to different types of subscriber attributes that are used for modelling and predicting customer churn. [6] applied Bayesian belief network to discover the most important features that have effects on customer churn in telecommunication industry. Similarly, association rules was used by [7] to extract features from original dataset with the goal of improving the performance of prediction while [8] developed a semi supervised data centric model to cluster high relevant and low redundant feature subset for churn prediction in feature engineering. In addition, [9] introduced a Relative Churn Fuzzy Feature Weight Model as an optimised feature subset for churn prediction. The method assigns fuzzy weights to attributes based on its relevance degree in the cluster as it builds clusters of interdependent relative associations between extracted features for effective churn prediction. By this, high precision and recall interdependent but uniquely weighted feature subset, which is sufficient for churn prediction are extracted. Thus, in reality, the preprocessed data are fed into machine learning algorithms alongside similar technics like customer segmentation for churn prediction.

Subsequently, as churn prediction methods continue to mitigate the demanding nature and high dimensionality issues present in the telecom dataset, [10] used logistic regression and decision tree model for churn prediction. In addition, a cutting-edge method via Support Vector Machine (SVM) algorithm using four kernel functions was introduced to predict the customer churn category by [11] in telecommunications. The research observed that SVM polynomial with four kernel functions outperformed three kernel functions in the prediction of customer churn. Likewise, [12] presented three Hybrid models in telecommunication sector for customer churn prediction. The designed approaches were based on efficient data clustering and prediction phase. In the analysis, the accuracy, precision and recall values was calculated and matched with other models like C5.0 and MLP-ANN for evaluation. Consequently, with the goal of improving customer churn in the telecom industry for market basket analysis, [13] presented a predictive approach that uses Decision Tree, KNearest Neighbor, Naïve Bayes and Random Forest to predict customer churn in Rapid Miner. However, as machine learning algorithms continue to dominate the churn prediction space, customer segmentation approach like Recency-Frequency-Monetary model have been used optimised for churn prediction [14] towards effective customer relationship management. The goal among others is to cluster customers according to how recent $(\mathrm{R})$ they have made a purchase, how frequent $(\mathrm{F})$ they make purchases, and how much, Monetary (M) they have bought. An algorithm for RFM sequential patterns generation using customers' purchasing data was implemented by [15] while [16] extended the RFM model by introducing the customers' influence degree (I). The goal was to harness the sociotransactional network behaviour of customers' through direct point to point relationship towards detecting the customer's power of influence via dependent to targeted customer RFM value analysis in the community. By this, churn misclassification was minimized and churn patterns improved significantly. Despite existing research works as documented, in section four, the developed blended churn predictive system is discussed. The objective of the system is to measure the Accuracy, Precision, Sensitivity of churn classification by harnessing the potency of RMFI churn category model and Ensemble Machine Learning approach through fuzzy weight features to generate four class of churn category i.e. Churner, Potential Churner, Inertia Customer and Premium customer, against existing Two churn categories (Churner and Non-Churner). By this, sample evaluation and experimental results of the research outcomes are presented in section four.

\section{Methodology: Blended Churn Predictive System}

The churn predictive system processes and analyses churn data by using the optimal feature set of customer records alongside customer behavioural segmentation. The developed model logic is represented in figure 1.

Where, $A_{f d}$ is the automatic feature possessing system, $R f_{f w}$ is the Relative Churn Fuzzy-Weight, $R F M I_{p}$ is the $C R F M I_{s}$ is the customer's RFMI score and $c c s_{i}$ is the customer churn score obtained from the general training and learning activities derived from customer's optimal feature analysis via ensemble machine learning. Thus, from figure 1, the churn predictive system performs the following operations:

1. Extract optimal features subset from the raw customer transaction dataset, 
2. Assign random fuzzy-weight to features for better churn classification,

3. Generate customer's churn score using ensemble model (Here, Adaboost and Random Forest),

4. Identify the influence of a customer in a community through socio-transaction network analysis,

5. Generate churn score for customers using the segment based behavioural analysis; here RFMI described in D and,
6. Find the variance of the two independent churn classification result for optimal churn classification.

Thus, from the processing stages, the blended churn predictive system was used to manipulate the raw transactional dataset in order to train and discover patterns that defines the churn category of a customer. The process also helped to define what feature triggers a churn class and when a customer churn may happen.

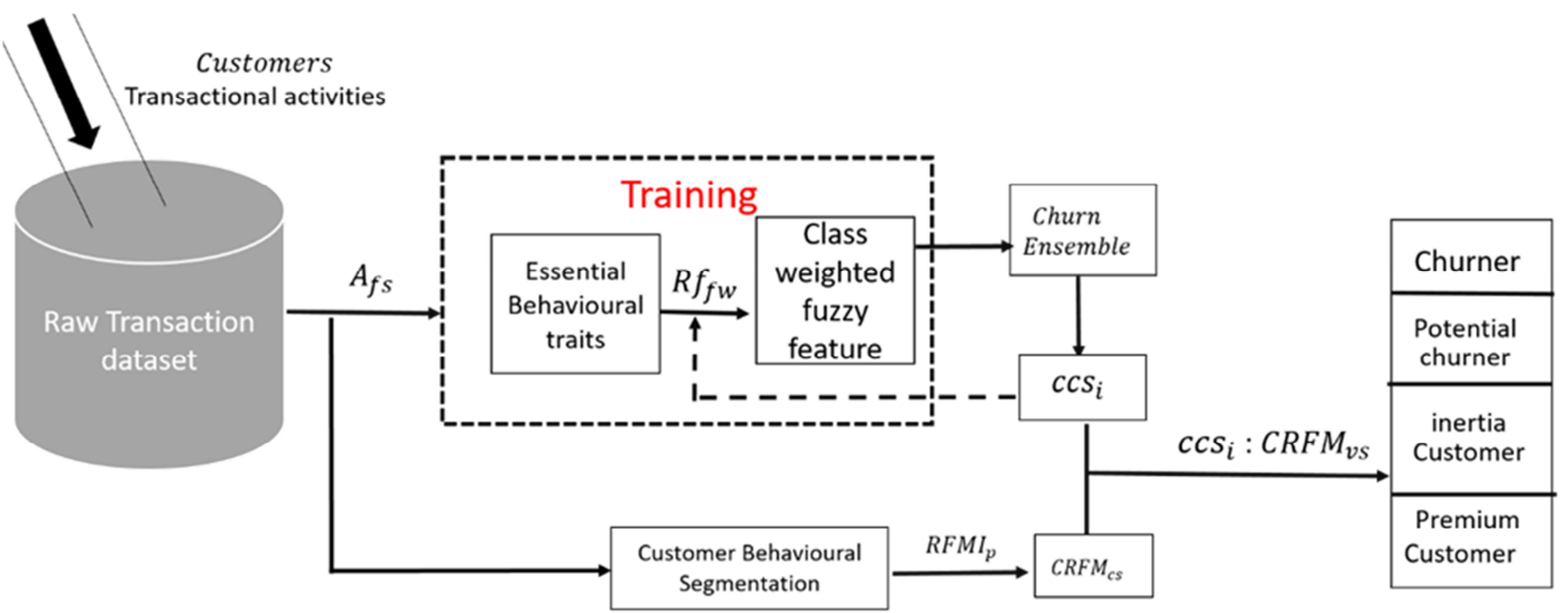

Figure 1. Churn Transaction Predictive System.

\section{Experiments and Evaluation}

In this section, we present a comparative result of the blended churn predictive system via the two ensemble machine learning models; Random Forest Ensemble in Table 1 and Adaboost Ensemble in Table 2. While the overall performance average analysis of the obtained results is also presented in figure 2 .

In figure 2, the average performance of Random Forest and Adaboost is presented.

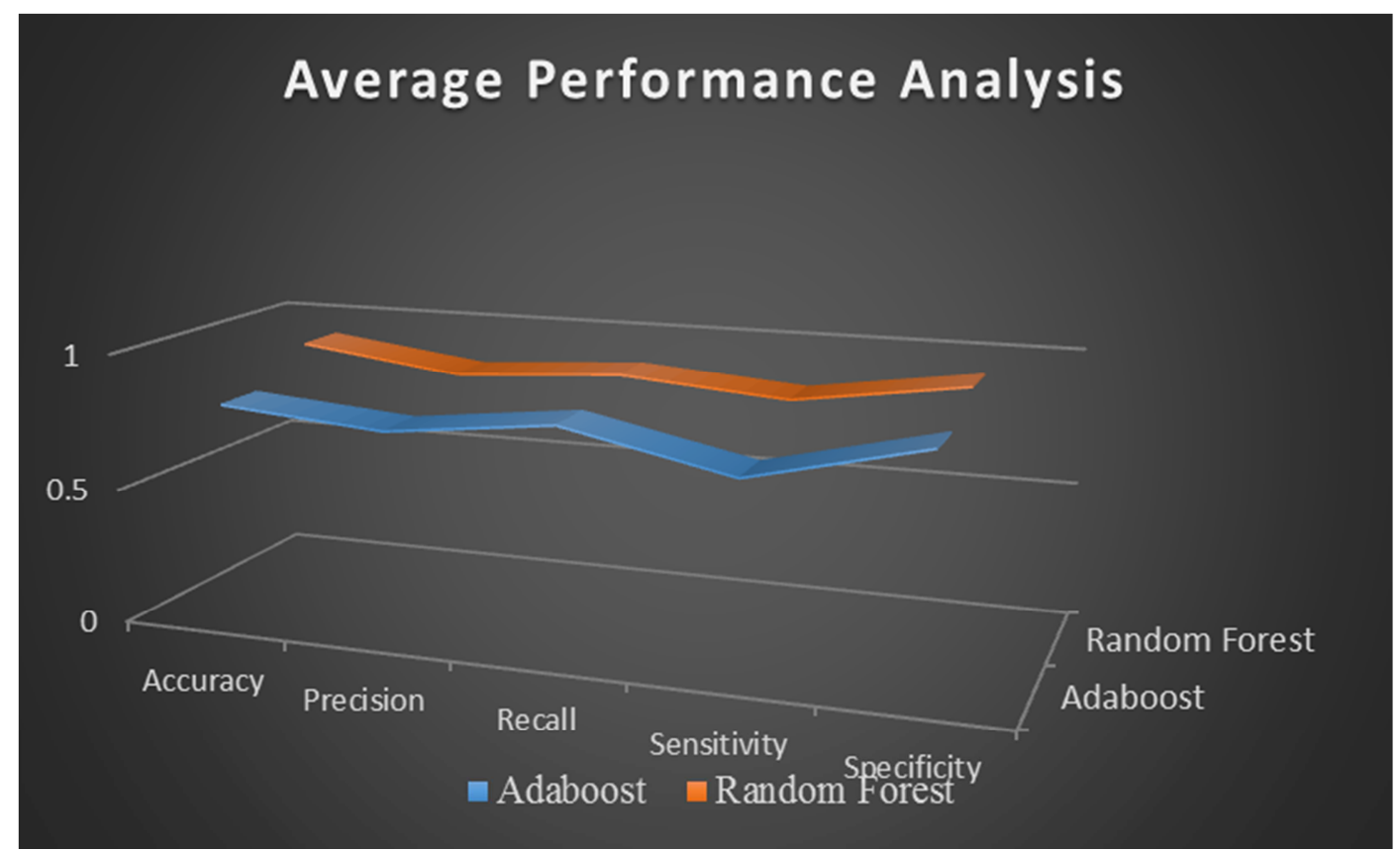

Figure 2. Average performance Analysis (Random Forest VS Adaboost). 
Table 1. Blended Churn Predictive System (BCPS) with Random Forest Ensemble.

\begin{tabular}{llllll}
\hline \multirow{2}{*}{ Churn Category } & \multicolumn{2}{l}{ Performance Metrics } & & & Sensitivity \\
\cline { 2 - 6 } & Accuracy & Precision & Recall & 0.83 & 0.90 \\
\hline Churner & 0.95 & 0.90 & 0.86 & 0.82 & 0.93 \\
Potential Churner & 0.88 & 0.82 & 0.92 & 0.81 & 0.91 \\
Inertia Customer & 0.87 & 0.78 & 0.80 & 0.83 & 0.92 \\
Premium Customer & 0.96 & 0.84 & 0.91 & \\
\hline
\end{tabular}

Table 2. Blended Churn Predictive System with Adaboost Ensemble.

\begin{tabular}{llllll}
\hline \multirow{2}{*}{ Churn Category } & Performance Metrics & & & \\
\cline { 2 - 6 } & Accuracy & Precision & Recall & Sensitivity & Specificity \\
\hline Churner & 0.81 & 0.88 & 0.78 & 0.55 & 0.90 \\
Potential Churner & 0.76 & 0.69 & 0.91 & 0.74 & 0.76 \\
Inertia Customer & 0.79 & 0.65 & 0.72 & 0.77 & 0.85 \\
Premium Customer & 0.83 & 0.76 & 0.84 & 0.67 & 0.82 \\
\hline
\end{tabular}

\section{Conclusion}

As consumer centric approach became more imperative in customer relationship management, the approach for churn prediction also needs to be enhanced for better performance. In this research, the blended churn predictive system provided a solution that helped to identify the market segment of a customers while augmenting the ascertained data with relevant customer behavioral patterns through ensemble learning for better churn classification. The system significantly reduced misclassification while improving customer churn categories from the existing binary class to a quadruple class of Churner, Potential Churner, Inertia Customer and Premium Customer respectively. The essence is to enhance targeted decision support for effective customer retention in customer behavior management.

\section{References}

[1] Azeem, M. and Usman, M., 2018. A fuzzy based churn prediction and retention model for prepaid customers in telecom industry. International Journal of Computational Intelligence Systems, 11 (1), pp. 66-78.

[2] Ahmad, A. K., Jafar, A. and Aljoumaa, K., 2019.“Customer churn prediction in telecom using machine learning in big data platform". Journal of Big Data, 6 (1), p. 28. Springer.

[3] Kiranjot Kaur and Sheveta Vashisht (2015) “ A Novel Approach for Providing the Customer Churn Prediction Model using Enhanced Boosted Trees Technique in Cloud Computing" International Journal of Computer Applications (0975 - 8887) Volume 114 - No. 7.

[4] Prashanth, R., Deepak, K. and Meher, A. K., 2017, July.“High accuracy predictive modelling for customer churn prediction in telecom industry". In International Conference on Machine Learning and Data Mining in Pattern Recognition (pp. 391402). Springer, Cham.

[5] X. Zhang, J. Zhu, S. Xu, and Y. Wan, (2012) "Predicting customer churn through interpersonal influence," Knowl.Based Syst., vol. 28, pp. 97-104, Apr. 2012.

[6] Pýnar Kisioglu, Y. Ilker Topcu, (2008) “Applying Bayesian Belief Network approach to customer churn analysis: A case study on the telecom industry of Turkey", Expert Systems with Applications vol. 38 7151-7157.

[7] Chih-Fong Tsai, Mao-Yuan Chen, (2015) "Variable selection by association rules for customer churn prediction of multimedia on demand", Expert Systems with Applications Vol 37.

[8] Ayodeji O. J IBITOYE and Olufade F. W ONIFADE (2016) "Intelligent Predictive Feature Selection Model for Effective Customer Churn Prediction" Proceedings of the 1st International Conference on Transition from Observation to Knowledge to Intelligence (TOKI 2016) held in University of Lagos, Nigeria during August 25-26, 2016. pp 231-239.

[9] Ayodeji O. J IBITOYE, Onifade F. W Onifade (2019)“Customer Churn Predictive Analytics using Relative Churn Fuzzy Feature-Weight Model in TelecomsInternational Journal of Information, Business and Management. Volume 11, Issue 3, pp 163-167.

[10] Guangli Nie, Wei Rowe, Lingling Zhang, Yingjie Tian, Yong Shi, (2011) "Credit card churn forecasting by logistic regression and decision tree", Expert Systems with Applications 38 15273-1528.

[11] Ionut Brandusoiu, Gavril Toderean, (2013) "Churn Prediction in the Telecommunications Sector Using Support Vector Machines".

[12] Amjad Hudaib, Reham Dannoun, Osama Harfoushi, Ruba Obiedat, Hossam Faris (2015) "Hybrid Data Mining Models for Predicting Customer Churn", J. Communications, Network and System Sciences, May 2015, 8, 91-96.

[13] Hanif, E., 2019. “Applications of data mining techniques for churn prediction and cross-selling in the telecommunications industry" (Doctoral dissertation, Dublin Business School).

[14] Sohrabi B, Khanlari A (2007). Customer lifetime value (CLV) measurement based on RFM model. Iranian Acc. Aud. Rev., 14 (47): 7-20.

[15] Cheng CH, Chen YS (2009). Classifying the segmentation of customer value via RFM model and RS theory. Expert. Syst. Appl., 36: 4176-4184.

[16] Ayodeji O. J IBITOYE, Onifade F. W Onifade Customers' Behavioural Management in Telecoms using SocioTransactional Influence on Recency-Frequency-Monetary (RFM) Approach in Churn Prediction -Springer International Journal of Information Technology- Accepted 2020. 